\title{
Maternal Outcome in women with placenta previa who had antepartum haemorrhage compared to those without haemorrhage: Retrospective Cohort Study
}

\author{
Philip Chung ${ }^{1}$, Kenny Cheer ${ }^{1}$, Eva Malacova ${ }^{2}$, Satomi Okan ${ }^{2}$, and Thangeswaran Rudra ${ }^{1}$ \\ ${ }^{1}$ Royal Brisbane and Women's Hospital \\ ${ }^{2}$ QIMR Berghofer
}

April 28, 2020

\begin{abstract}
Objective: To compare maternal outcomes for placenta praevia (PP) between those who experience antepartum haemorrhage (APH) and those without APH. Design: Retrospective cohort study. Setting: Royal Brisbane \& Women's Hospital, Australia. Population: Over ten years, a total of 368 women with placenta praevia (PP) were recruited and divided into APH ( $\mathrm{n}=230$ ) and No APH $(\mathrm{n}=138)$. Methods: Data retrieved from institution-based obstetric database were analysed using logistic regressions for binary outcomes and Poisson or negative binomial regressions for counts. Main Outcome Measures: Type of $\mathrm{PP}$, timing and mode of delivery, intra-operative and postpartum complications, volume of blood loss and number of blood transfusions. Results: Compared to women without APH, women who experienced APH had a greater proportion of major $\mathrm{PP}(\mathrm{OR}=2.88, \mathrm{p}<0.001)$, emergency lower uterine segment $(\mathrm{OR}=6.24, \mathrm{p}<0.001)$ and classical $(\mathrm{OR}=14.9, \mathrm{p}<0.001)$ sections, use of general anaesthesia $(\mathrm{OR}=3.14, \mathrm{p}<0.001)$, preterm delivery (median 35.4 vs 38.0 weeks, $\mathrm{p}<0.001)$. There was no significant differences intra-operatively (number of uterotonics used or additional surgical techniques), greater volume of bloods loss $(\operatorname{IRR}=1.20, \mathrm{p}=0.006)$, increased frequency of blood transfusion ( $\mathrm{IRR}=3.36, \mathrm{p}<0.001)$, and longer post-operative hospital stay $(\mathrm{IRR}=1.27, \mathrm{p}=0.001)$. There was a total of four hysterectomies and no intensive care unit admissions or maternal deaths. Conclusions: Women with PP who experienced APH had a tendency for preterm delivery, emergency caesarean section, greater blood loss requiring more blood transfusion and longer hospital admissions. However, a lack of serious maternal morbidity among these women provides support for an individualised approach towards management of bleeding in PP. Funding: No funding body. Keywords: Antepartum Haemorrhage, Placenta Praevia, maternal outcomes.
\end{abstract}

\section{TWEETABLE ABSTRACT}

Bleeding in placenta praevia makes no difference in terms of serious intra-operative or postpartum maternal outcomes.

\section{INTRODUCTION}

One of the most important causes of antepartum haemorrhage (APH) is Placenta praevia (PP), which is characterised by abnormal placentation in close proximity to the internal cervical os, with a reported prevalence of 5 per 1000 pregnancies worldwide. ${ }^{1}$ The Royal College of Obstetricians and Gynaecologists $(\mathrm{RCOG})^{2}$ defines APH as bleeding from or in to the genital tract beyond 24 weeks of pregnancy and acknowledges that there is no consistent definition for describing its severity. However, APH is globally regarded as a leading cause of perinatal and maternal mortality, complicating $3-5 \%$ of pregnancies. ${ }^{3}$

Compared to non-placenta praevia women, those with placenta praevia have approximately a four- to tenfold increased risk of APH. ${ }^{4,5}$ In 2017, a systematic review of 29 studies by Fan et al. ${ }^{6}$ reported that among women with PP the overall prevalence of APH was $51.6 \%$, ranging from $20 \%$ to $90 \%{ }^{7,8}$ Women with PP who 
experience increasing episodes of APH have been associated with greater risks of requiring blood transfusion, preterm caesarean section, and emergency hysterectomy. ${ }^{9}$

Previously, RCOG has recommended that women with PP and previous bleeding events require admission at or after 34 weeks. However, the most recent RCOG (2018) guidelines ${ }^{2}$ recommend that women with recurrent bleeding be given tailored antenatal care based on recommendations from a Cochrane systematic review ${ }^{10}$ that showed no clear disadvantage to a policy of home versus hospital care. Likewise, women with PP without APH can be managed in the outpatient setting with similar outcomes compared to hospitalisation. ${ }^{11}$

Although APH is common in women with PP, it has not been extensively evaluated in the literature, with previous studies recruiting small populations of less than 250 cases. ${ }^{12-14}$ The aim of this large scale retrospective study was to examine a range of obstetric outcomes for women with placenta praevia complicated by any bleeding episodes compared to those without.

\section{METHODS}

\section{Study design, study population and data collection}

This retrospective cohort study included all women who were diagnosed with placenta praevia at the time of their delivery at the Royal Brisbane \& Women's Hospital, one of the largest tertiary referral unit in Queensland, between $1^{\text {st }}$ January 2009 and $31^{\text {st }}$ December 2018. Women diagnosed with placenta accreta spectrum disorders $(n=65)$ and duplicate records for those who gave birth to twins $(n=11)$ were excluded, thus leaving 368 women for the analysis. This research was approved by the institutional Human Research Ethics Committee (LNR/2018/QRBW/43114). Data was retrieved via our institution-based obstetric database and review of patient charts. Surgeon operation notes and anaesthetic documentation were also carefully examined during the data retrieval period.

\section{Exposure}

The diagnosis of PP was made after 34 weeks of pregnancy by ultrasound or at time of delivery. APH was defined by any episode of bleeding from the genital tract from beyond 24 weeks of gestation.

\section{Core Outcome Sets}

The main maternal outcomes investigated included the type of PP, timing and mode of delivery, use of general anaesthesia, intraoperative complications and any measures used to prevent and control significant bleeding, such as the type and number of uterotonics expended, additional surgical techniques and transfusions. The total volume of blood loss and postpartum complications including the hospital stay post-delivery were also recorded.

\section{Other variables}

Information was available on maternal demographics included maternal age, parity, maternal ethnicity, maternal smoking, IVF, a history of caesarean section and a history of previous surgery.

\section{Statistical analysis}

Means and standard deviations were used to summarise normally distributed variables and compared using a t-test, while medians and interquartile ranges were used and compared using a Mann-Whitney $U$ test for non-normally distributed variables. Categorical variables were summarised as counts and percentages, and compared using a Chi-squared test. To explore the association between APH and the various obstetrics outcomes, we used logistic, negative binomial and Poisson regression as appropriate. All analyses were conducted using STATA version 15.1 (Stata Corporation, College Station, Texas, USA).

\section{RESULTS}

We investigated medical records of 368 women who were diagnosed with PP from 2009 to 2018. Of these, two thirds $(63 \%)$ had APH, of whom $66 \%$ had major praevia. Women with APH had greater odds of major $\mathrm{PP}(\mathrm{OR}=2.77,95 \% \mathrm{CI}: 1.79-4.28, p<0.001)$. Women with APH were on average 1.4 years younger than 
women without $(\mathrm{p}=0.01)$ (Table 1$)$. There was no significant difference between women with and without APH in the mean body mass index, maternal ethnicity, maternal smoking, parity, a history of caesarean section (CS), a history of uterine surgery, and IVF.

$<$ Table $1>$

The percentage of emergency (both classical and lower segment) CS, and use of general anaesthetic was greater among women with APH compared to women without (Table 2). Compared to women without $\mathrm{APH}$, women with APH had greater risk of emergency classical CS relative to elective LSCS $(\mathrm{RRR}=14.9$, 95\% CI: 3.37-66.0). Women with APH had greater odds general anaesthetics (OR=3.14, 95\% CI: 1.90$5.18)$, and extension of uterine tear $(\mathrm{OR}=3.67,95 \%$ CI: 0.44-30.81), though this did not reach statistical significance. The median timing of delivery for women with APH (35.4 weeks) was significantly earlier than for women without APH (38.0 weeks) (Table 2). Women with APH had a higher median estimated blood loss, as reflected by the IRR=1.22 (95\% CI: 1.06-1.39).

$<$ Table $2>$

The percentage of medical measures was greater among women with APH (syntocinon bolus and infusion) and lower for carbetocin, compared to women without APH (Table 3a). Women with APH had higher odds of syntocinon bolus ( $\mathrm{OR}=1.83,95 \%$ CI: 1.14-2.91) and infusion $(\mathrm{OR}=1.98,95 \%$ CI: 1.19-3.31), but lower odds of carbetocin (OR=0.27, 95\% CI: 0.12-0.59). There was no association of APH with the different types of transfusions and with a higher number of uterotonics (Table 3a). Overall, women with APH had a higher percentage of additional surgical techniques compared to women without, most notably for surgicell $(\mathrm{OR}=3.43,95 \%$ CI: 1.39-8.44) and Bakri balloon (OR=10.2, 95\% CI: 1.34-78.1) (Table 3b). There were 3 women with $\mathrm{APH}$ and 1 without $\mathrm{APH}$ who underwent hysterectomy. As expected, more packed RBCs were used for women with APH (IRR=3.76, 95\% CI: 1.96-7.21) (Table 3c). Women with APH had slightly greater odds of any postpartum complication ( $\mathrm{OR}=1.67,95 \%$ CI: 1.08-2.59) and longer length of post-op hospital stay (IRR=1.28, 95\% CI: 1.12-1.47) (Table 4). There were no ICU admissions or maternal deaths recorded.

$<$ Table 3>

$<$ Table $4>$

\section{DISCUSSION}

Main Findings

Our study found that APH in the setting of PP has significant implications for mothers intraoperatively and postpartum, including a tendency for preterm emergency caesarean sections under general anaesthesia, with greater blood loss requiring transfusion, and longer postpartum stay.

The incidence of PP in our population was $0.8 \%$, compared to the incidence reported in the current literature at $0.2-0.4 \%{ }^{8,15}$ This is likely due to the large number of high-risk obstetric referrals received at the study hospital known to be a major tertiary centre.

In our study, the majority $(62 \%)$ of women with placenta praevia experienced antenatal haemorrhage, which is consistent with a previous study ${ }^{14}$ and slightly greater than reported $(51.6 \%)$ within the systematic review and meta-analysis of twenty-nine articles with 4687 individuals by Fan et al. ${ }^{6}$ Despite excluding all placenta accreta cases, our study examines the largest number of women diagnosed solely with placenta praevia compared to any previous individual article noted by Fan et al. ${ }^{6}$

We found that maternal characteristics, though may predispose a diagnosis of placenta praevia, made no difference in risk of antepartum bleeding in women with placenta praevia, alike a prior study by Mastrolia et al. ${ }^{16}$ However, the diagnosis of major PP significantly increases the risk of APH (OR $2.88 p<0.001$ ) compared to minor PP. This result is supported by another large retrospective cohort of 306 women by Bahar et al. ${ }^{17}$ (OR 3.18, 95\% CI 1.58-6.4, $p=0.001$ ) and similarly reported in a population of 121 women by Bhide et al. ${ }^{18}$ comparing APH in major versus minor PP $(57.1 \%$ versus $47.5 \% p<0.05)$. Besides the 
degree of PP, developing research suggests that the likelihood of APH might also be related to the placental edge thickness ${ }^{19}$ and echo-free space in the lower edge of the placenta. ${ }^{20}$

The increased risk of preterm delivery in women with PP is well established..$^{21,22}$ In our study, women with PP who experienced bleeding were significantly more likely to deliver earlier than those without bleeding (median 35.4 versus 38.0 weeks, $p<0.001$ ). This finding is consistent with results by Lam et al., ${ }^{14}$ who also found that newborns delivered from mothers with APH were smaller and more frequently required nursery admission. Therefore the earlier timing of delivery in placenta praevia seems to correlate with the incident of at least one bleeding episode and degree of PP, which in itself increases the tendency to bleed.

Pivano et al. ${ }^{23}$ published a scoring system that predicted the risk of emergency caesarean for women with placenta praevia based on the type of PP, frequency and intensity of antenatal bleeding and gestational age at sentinel bleed. In our population, women who had experienced APH were more likely to undergo an emergency caesarean sections than their asymptomatic counterparts for lower uterine segment (61\% vs $25 \%$ ) and classical incisions ( $8.5 \%$ vs $1.4 \%$ ), which correlates well with significant findings by Love et al. ${ }^{24}(63 \%$ vs $25 \% p<0.001$ ) and Fishman et al. ${ }^{25}$ (OR $17.795 \%$ CI 6.1-51.7).

Interestingly, in our study there was a novel finding of three-fold increased risk of undergoing general anaesthesia in women with bleeding that has not been reported before in the literature. This might be explained by the urgency of delivery as suggested by earlier gestations and greater proportion of emergency caesareans in the antepartum bleeding cohort. The use of general anaesthesia in caesarean sections is associated with a greater volume of blood loss than neuroaxial anaesthesia. ${ }^{26}$

We found that both syntocinon bolus and infusions were used more frequently intra-operatively in situations where bleeding had occurred. Otherwise, there was no difference in the median number of uterotonics used between the compared cohorts. Likewise, besides use of surgicell (OR 3.56, 95\% CI 1.45-8.73) and Bakri balloon (OR 10.3, 95\% CI 1.35-78.2), there were no differences in additional surgical techniques required for managing bleeding in the APH group versus those without APH. A plausible explanation for the lack of statistical significance in both medical and additional surgical techniques, including hysterectomy, might be the heterogeneity of surgical and anaesthetic experience and individual preferences within the study hospital, which may range from junior obstetric trainees to skilled specialists.

Women with PP who bled antenatally were likely to have significantly greater volume of blood loss postoperatively than their asymptomatic counterparts (IRR 1.20, 95\% CI 1.05-1.37), requiring over three-fold the number of blood transfusions. Mastrolia et al. ${ }^{16}$ similarly found a tendency for blood transfusions in the presence of APH, unlike previous studies. ${ }^{14,24}$

Besides a marginally longer postpartum hospital stay for those who experienced APH, there was no difference in postoperative complications. The current literature has scarcely focused on postpartum maternal outcomes in $\mathrm{PP},{ }^{27,28}$ and for those that have evaluated this aspect have also found no significant difference between the presence and absence of APH. ${ }^{14}$

Strength and limitations of the study

A major strength of this retrospective population-based study was that we were able to analyse a rare condition over a period of ten years and therefore we were able to provide a true representation of placenta praevia outcomes.

The present study has been conducted within a major referral and tertiary hospital and thus we were able to recruit over 350 patients. Additionally, our hospital is well resourced both medically and surgically, with maternal-fetal-medicine obstetricians, gynae-oncology specialists and interventional radiologists on standby to optimise maternal care. Our results are widely generalisable because the study hospital is representative of the type of tertiary centres where the majority of these rare high-risk placenta praevia cases are referred.

The perinatal database utilised in this study is obtained from obstetrician documentation and routinely undergoes large institution-based rigorous examination for accuracy to mitigate risk of misclassification and 
recall bias, which are intrinsic limitations associated with retrospective studies.

The authors acknowledge that though clinical practices may change over the years with new clinical insights, it is unlikely to substantially influence the results as effects would be equally distributed between the comparison cohorts.

\section{Interpretation}

Overall, our results demonstrate that any bleeding episode during the antenatal period for a woman diagnosed with placenta praevia should be taken into serious consideration. Compared to women who remain asymptomatic during their pregnancy, those who experience APH have increased risks of blood loss requiring blood transfusion, preterm delivery, emergency caesarean section and general anaesthesia, as well as longer postpartum stay.

\section{CONCLUSION}

There are only a few differences in regards to intra-operative management and post-operative complications, none of which pose serious maternal morbidity. Therefore our findings further strengthen the RCOG recommendations of an individualised approach towards managing placenta praevia with any bleeding episode. We advise taking into account patients' wishes, their ability to access emergency services and the availability of transportation. Hence, our findings suggest that it may be reasonable to continue safely managing women with placenta praevia and previous bleeding episodes in the outpatient setting.

Further large-scale, multicentre prospective studies using a single validated method of data collection is encouraged to provide a more accurate representation of maternal outcomes directly linked to placenta praevia. In order to optimise maternal outcomes in placenta praevia further research ought to focus on the aetiology of $\mathrm{APH}$ and formulate strategies for the prevention and treatment of bleeding.

\section{ACKNOWLEDGEMENTS}

Nil.

\section{DISCLOSURE OF INTEREST}

The authors have declared that no financial, personal, political, intellectual or religious interests exist. CONTRIBUTION TO AUTHORSHIP

P.C. Conception, Writing - original draft preparation, Data Retrieval and Interpretation K.C. Data Retrieval E.M.Writing - Reviewing and Editing, Data Interpretation S.O.Reviewing and Editing, Data Analysis and Interpretation T.R.Conception, Supervision, Planning

\section{DETAILS OF ETHICS APPROVAL}

Royal Brisbane and Women's Hospital Human Research Ethics Committee, approved 30 August 2018, reference number: LNR/2018/QRBW/43114

\section{FUNDING}

The authors have received no specific funding for this work.

\section{REFERENCES}

1. Cresswell JA, Ronsmans C, Calvert C, Filippi V. Prevalence of placenta praevia by world region: a systematic review and meta-analysis. Tropical medicine \& international health. 2013 Jun;18(6):712-24.

2. Jauniaux ER, Alfirevic Z, Bhide AG, Belfort MA, Burton GJ, Collins SL, et al. Placenta praevia and placenta accreta: diagnosis and management. BJOG: An International Journal of Obstetrics \& Gynaecology. 2018.

3. Calleja-Agius J, Custo R, Brincat MP, Calleja N. Placental abruption and placenta praevia. European Clinics in Obstetrics and Gynaecology. 2006 Nov 1;2(3):121-7. 
4. Rosenberg T, Pariente G, Sergienko R, Wiznitzer A, Sheiner E. Critical analysis of risk factors and outcome of placenta previa. Archives of gynecology and obstetrics. 2011 Jul 1;284(1):47-51.

5. Crane JM, Van den Hof MC, Dodds L, Armson BA, Liston R. Maternal complications with placenta previa. American journal of perinatology. 2000;17(02):101-6.

6. Fan D, Wu S, Liu L, Xia Q, Wang W, Guo X, et al. Prevalence of antepartum hemorrhage in women with placenta previa: a systematic review and meta-analysis. Scientific reports. 2017 Jan 9;7:40320.

7. Vergani P, Ornaghi S, Pozzi I, Beretta P, Russo FM, Follesa I, et al. Placenta previa: distance to internal os and mode of delivery. American journal of obstetrics and gynecology. 2009 Sep 1;201(3):266e1.

8. Tuzovic L. Complete versus incomplete placenta previa and obstetric outcome. International Journal of Gynecology \& Obstetrics. 2006 May;93(2):110-7.

9. Ruiter L, Eschbach SJ, Burgers M, Rengerink KO, Van Pampus MG, Van Der Goes BY, et al. Predictors for emergency cesarean delivery in women with placenta previa. American journal of perinatology. 2016 Dec;33(14):1407-14.

10. Neilson JP. Interventions for suspected placenta praevia. Cochrane Database of Systematic Reviews. 2003(2).

11. Silver RM. Abnormal placentation: placenta previa, vasa previa, and placenta accreta. Obstetrics \& Gynecology. 2015 Sep 1;126(3):654-68.

12. Hill DJ, Beischer NA. Placenta praevia without antepartum haemorrhage. Australian and New Zealand Journal of Obstetrics and Gynaecology. 1980 Feb;20(1):21-3.

13. Rosen DM, Peek MJ. Do women with placenta praevia without antepartum haemorrhage require hospitalization?. Australian and New Zealand journal of obstetrics and gynaecology. 1994 May;34(2):130-4.

14. Lam M, Wong SF, Chow KM, Ho LC. Women with placenta praevia and antepartum haemorrhage have a worse outcome than those who do not bleed before delivery. Journal of obstetrics and Gynaecology. 2000 Jan 1;20(1):27-31.

15. Lal AK, Hibbard JU. Placenta previa: an outcome-based cohort study in a contemporary obstetric population. Archives of gynecology and obstetrics. 2015 Aug 1;292(2):299-305.

16. Mastrolia SA, Baumfeld Y, Loverro G, Yohai D, Hershkovitz R, Weintraub AY. Placenta previa associated with severe bleeding leading to hospitalization and delivery: a retrospective population-based cohort study. The Journal of Maternal-Fetal \& Neonatal Medicine. 2016 Nov 1;29(21):3467-71.

17. Bahar A, Abusham A, Eskandar M, Sobande A, Alsunaidi M. Risk factors and pregnancy outcome in different types of placenta previa. Journal of Obstetrics and Gynaecology Canada. 2009 Feb 1;31(2):126-31.

18. Bhide A, Prefumo F, Moore J, Hollis B, Thilaganathan B. Placental edge to internal os distance in the late third trimester and mode of delivery in placenta praevia. BJOG: An International Journal of Obstetrics \& Gynaecology. 2003 Sep;110(9):860-4.

19. Ghourab S. Third-trimester transvaginal ultrasonography in placenta previa: does the shape of the lower placental edge predict clinical outcome?. Ultrasound in Obstetrics and Gynecology: The Official Journal of the International Society of Ultrasound in Obstetrics and Gynecology. 2001 Aug;18(2):103-8.

20. Saitoh M, Ishihara K, Sekiya T, Araki T. Anticipation of uterine bleeding in placenta previa based on vaginal sonographic evaluation. Gynecologic and obstetric investigation. 2002;54(1):37-42.

21. Ananth CV, Demissie K, Smulian JC, Vintzileos AM. Relationship among placenta previa, fetal growth restriction, and preterm delivery: a population-based study. Obstetrics \& Gynecology. 2001 Aug 1;98(2):299-306.

22. Zlatnik MG, Cheng YW, Norton ME, Thiet MP, Caughey AB. Placenta previa and the risk of preterm delivery. The Journal of Maternal-Fetal \& Neonatal Medicine. 2007 Jan 1;20(10):719-23.

23. Pivano A, Alessandrini M, Desbriere R, Agostini A, Opinel P, d'Ercole C, et al. A score to predict the risk of emergency caesarean delivery in women with antepartum bleeding and placenta praevia. European Journal of Obstetrics \& Gynecology and Reproductive Biology. 2015 Dec 1;195:173-6.

24. Love CD, Fernando KJ, Sargent L, Hughes RG. Major placenta praevia should not preclude outpatient management. European Journal of Obstetrics \& Gynecology and Reproductive Biology. 2004 
Nov 10;117(1):24-9.

25. Fishman SG, Chasen ST, Maheshwari B. Risk factors for preterm delivery with placenta previa. Journal of perinatal medicine. 2012 Jan 1;40(1):39-42.

26. Heesen M, Hofmann T, Kloehr S, Rossaint R, van de Velde M, Deprest J, et al. Is general anaesthesia for caesarean section associated with postpartum haemorrhage? Systematic review and meta-analysis. Acta Anaesthesiologica Scandinavica. 2013 Oct;57(9):1092-102.

27. Gibbins KJ, Einerson BD, Varner MW, Silver RM. Placenta previa and maternal hemorrhagic morbidity. The Journal of Maternal-Fetal \& Neonatal Medicine. 2018 Feb 16;31(4):494-9.

28. Meena N, Dave A, Meena S, Meena A, Shrivastava A. Impact of placenta praevia on obstetric outcome. Int J Reprod Contracept Obstet Gynecol. 2015;4(1):76-8o.

\section{Hosted file}

Placenta Praevia APH vs No APH Tables March 2020 BJOG.docx available at https://authorea.com/ users/311892/articles/442539-maternal-outcome-in-women-with-placenta-previa-who-had-antepartumhaemorrhage-compared-to-those-without-haemorrhage-retrospective-cohort-study 